\title{
Uniqueness of Meromorphic Functions Sharing One Value and a Small Meromorphic Function
}

\author{
Subhas.S.Bhoosnurmath ${ }^{1}$, K.S.L.N.Prasad ${ }^{2}$ \\ ${ }^{I}$ Department of Mathematics, Karnatak University,Dharwad-580003-INDIA \\ ${ }^{2}$ Department of Mathematics, Karnatak Arts College, Dharwad-580001-INDIA
}

Abstract: In this paper we prove a uniqueness theorem for a meromorphic function which is sharing one value and a small meromorphic functionwith its derivatives.

\section{Introduction}

Let $f$ and $g$ be two non-constant meromorphic functions defined on the complex plane. If $f$ and $g$ have the same a-points with the same multiplicities, we say that $\mathrm{f}$ and $\mathrm{g}$ share the value a $\mathrm{CM}$ (counting multiplicities).

We wish to list few results which are already proved.

Rubel and C. C. Yang have proved the following result.

Theorem A[1]: Let $\mathrm{f}$ be a non constant entire function. If $\mathrm{f}$ and $\mathrm{f}$ ' share two finite, distinct values $\mathrm{CM}$, then $\mathrm{f} \equiv \mathrm{f}$ Later, Mues and Steinmetz improved Theorem A with the following result.

Theorem $B$ [2]: Let $\mathrm{f}$ be a non constant entire function. If $\mathrm{f}$ and $\mathrm{f}^{\prime}$ share two finite distinct values IM, then $\mathrm{f} \equiv$ $f^{\prime}$.

Further, Jank, Mues and Volkmann proved the following two results in [3]

Theorem C: Let $\mathrm{f}$ be a non constant meromorphic function and let $\mathrm{a} \neq 0$ be a finite constant. If $\mathrm{f}, \mathrm{f}^{\prime}$ and $\mathrm{f}^{\prime \prime}$ share the value a $\mathrm{CM}$, then $\mathrm{f} \equiv \mathrm{f}^{\prime}$.

Theorem D: Let $\mathrm{f}$ be a non constant entire function and let $\mathrm{a} \neq 0$ be a finite constant. If $\mathrm{f}$ and $\mathrm{f}^{\prime}$ share the value a IM and if $\mathrm{f}^{\prime \prime}(\mathrm{z})=\mathrm{a}$, whenever $\mathrm{f}(\mathrm{z})=\mathrm{a}$ then $\mathrm{f} \equiv \mathrm{f}^{\prime}$.

We wish to consider a slightly different case where a meromorphic

function share one value and a small meromorphic function.

Our main result is the following.

Theorem: Let $\mathrm{f}$ be a non constant meromorphic function with $\mathrm{N}(\mathrm{r}, \mathrm{f})+\mathrm{N}\left(\mathrm{r}, \frac{1}{\mathrm{f}}\right)=\mathrm{S}(\mathrm{r}, \mathrm{f})$. Let $\chi$ be a small meromorphic function satisfying $\quad \mathrm{T}(\mathrm{r}, \chi)=\mathrm{o}\{\mathrm{T}(\mathrm{r}, \mathrm{f})\}$.

If $\mathrm{f}$ and $\mathrm{f}$ ' share $\infty$ and $\chi \mathrm{CM}$ and satisfies the equation

$$
\mathrm{kf}^{\prime}-\mathrm{f}-(\mathrm{k}-1) \chi=0
$$

for $\mathrm{k} \neq 0$, then $\mathrm{f} \equiv \mathrm{f}^{\prime}$.

Further, if $\mu$ and $\lambda$ are two small meromorphic functions satisfying $\mathrm{T}(\mathrm{r}, \mu)=\mathrm{o}\{\mathrm{T}(\mathrm{r}, \mathrm{f})\}$ and $\mathrm{T}(\mathrm{r}, \lambda)=\mathrm{o}\{\mathrm{T}(\mathrm{r}, \mathrm{f})\}(\chi \neq \mu, \chi \neq \lambda)$ satisfying

$\bar{N}\left(r, \frac{1}{f-\mu}\right)+N\left(r, \frac{1}{f-\lambda}\right)+\bar{N}(r, f)=S(r, f)$, then, $\frac{f-\mu}{\chi-\mu}=\frac{f^{\prime}-\lambda}{\chi-\lambda}$.

We require the following Lemmas to prove our result.

Lemma 1 [4] Let $\mathrm{f}$ be a non constant meromorphic function. Then,

for $\mathrm{n} \geq 1$,

$$
\mathrm{N}\left(\mathrm{r}, \frac{1}{\mathrm{f}^{(\mathrm{n})}}\right) \leq 2^{\mathrm{n}-1}\left[\overline{\mathrm{N}}\left(\mathrm{r}, \frac{1}{\mathrm{f}}\right)+\overline{\mathrm{N}}(\mathrm{r}, \mathrm{f})\right]+\mathrm{N}\left(\mathrm{r}, \frac{1}{\mathrm{f}}\right)+\mathrm{S}(\mathrm{r}, \mathrm{f}) .
$$

Lemma 2 [4]: Let $f_{1}$ and $f_{2}$ be two non constant meromorphic functions and $\alpha_{1} \not \equiv 0, \alpha_{2} \not \equiv 0$ be two small meromorphic functions satisfying $\mathrm{T}\left(\mathrm{r}, \alpha_{\mathrm{i}}\right)=\mathrm{o}\{\mathrm{T}(\mathrm{r}, \mathrm{f})\}(\mathrm{i}=1,2)$, where $\mathrm{T}(\mathrm{r}, \mathrm{f})=\operatorname{Max}\left\{\mathrm{T}\left(\mathrm{r}, \mathrm{f}_{1}\right), \mathrm{T}\left(\mathrm{r}, \mathrm{f}_{2}\right)\right\}$. 


$$
\text { If } \alpha_{1} f_{1}+\alpha_{2} f_{2} \equiv 1 \text {, then, } \quad T\left(r, f_{1}\right)<\bar{N}\left(r, \frac{1}{f_{1}}\right)+\bar{N}\left(r, \frac{1}{f_{2}}\right)+\bar{N}\left(r, f_{1}\right)+o\{t(r, f)\}
$$

\section{Proof of the Theorem}

From (1), we have $\mathrm{kf}^{\prime}-\mathrm{f}-(\mathrm{k}-1) \chi=0$

Therefore, $\frac{\mathrm{f}-\chi}{\mathrm{f}^{\prime}-\chi}=\mathrm{k}$, where $\mathrm{k}$ is a non zero constant.

Put $\mathrm{f}_{1}=\frac{1}{\chi} \mathrm{f}, \mathrm{f}_{2}=\mathrm{k}, \mathrm{f}_{3}=\frac{-\mathrm{k}}{\chi} \mathrm{f}^{\prime} \quad($ where $\chi \neq 0)$ so that $\mathrm{f}_{1}+\mathrm{f}_{2}+\mathrm{f}_{3} \equiv 1$

If $\mathrm{k} \neq 1$, we get, $\frac{1}{\chi(1-\mathrm{k})} \mathrm{f}-\frac{\mathrm{k}}{\chi(1-\mathrm{k})} \mathrm{f}^{\prime} \equiv 1$

Then, by Lemma 2, we have

$$
\begin{array}{r}
\mathrm{T}(\mathrm{r}, \mathrm{f})<\overline{\mathrm{N}}\left(\mathrm{r}, \frac{1}{\mathrm{f}}\right)+\overline{\mathrm{N}}\left(\mathrm{r}, \frac{1}{\mathrm{f}^{\prime}}\right)+\overline{\mathrm{N}}(\mathrm{r}, \mathrm{f})+\mathrm{S}(\mathrm{r}, \mathrm{f}) \\
\text { and } \mathrm{T}\left(\mathrm{r}, \mathrm{f}^{\prime}\right)<\overline{\mathrm{N}}\left(\mathrm{r}, \frac{1}{\mathrm{f}}\right)+\overline{\mathrm{N}}\left(\mathrm{r}, \frac{1}{\mathrm{f}^{\prime}}\right)+\overline{\mathrm{N}}\left(\mathrm{r}, \mathrm{f}^{\prime}\right)+\mathrm{S}(\mathrm{r}, \mathrm{f}) \text {. }
\end{array}
$$

Using Lemma 1 and noting that $N\left(r, f^{(k)}\right)=N(r, f)+k \bar{N}(r, f)$,

we get ,

$$
\begin{array}{r}
T(r, f) \leq 3 N\left(r, \frac{1}{f}\right)+2 N(r, f)+S(r, f) \\
\text { and } T\left(r, f^{\prime}\right) \leq 3 N\left(r, \frac{1}{f}\right)+3 N(r, f)+S(r, f)
\end{array}
$$

Adding (3) and (4) we get

$$
\begin{aligned}
T(r, f)+T\left(r, f^{\prime}\right) & \leq 6 N\left(r, \frac{1}{f}\right)+5 N(r, f)+S(r, f) \\
& \leq 6\left[N(r, f)+N\left(r, \frac{1}{f}\right)\right]+S(r, f)
\end{aligned}
$$

This gives $T(r, f)+T\left(r, f^{\prime}\right) \leq S(r, f)$ in view of the hypothesis.

$$
\text { Or } 1 \leq \frac{\mathrm{S}(\mathrm{r}, \mathrm{f})}{\mathrm{T}(\mathrm{r}, \mathrm{f})+\mathrm{T}\left(\mathrm{r}, \mathrm{f}^{\prime}\right)} \rightarrow 0 \text {, as } \mathrm{r} \rightarrow \infty
$$

Or $1 \leq 0$, which is a contradiction.

This contradiction proves that $\mathrm{k}=1$.

Therefore, $\frac{\mathrm{f}-\chi}{\mathrm{f}^{\prime}-\chi}=1$

$$
\begin{aligned}
& \text { Or } \mathrm{f}-\chi=\mathrm{f}^{\prime}-\chi \\
& \text { Or } \mathrm{f} \equiv \mathrm{f}^{\prime} .
\end{aligned}
$$

Further, $f-\mu=\left(f^{\prime}-\lambda\right)+(\lambda-\mu)$.

$$
\text { If } \lambda \neq \mu \text { then } \frac{\mathrm{f}-\mu}{\lambda-\mu}-\frac{\mathrm{f}^{\prime}-\mu}{\lambda-\mu}=1
$$

Since $T(r, f) \leq T(r, f-\mu)+o\{T(r, f)\}$.

By Lemma 6, we have 


$$
\begin{array}{r}
\mathrm{T}(\mathrm{r}, \mathrm{f})<\overline{\mathrm{N}}\left(\mathrm{r}, \frac{1}{\mathrm{f}-\mu}\right)+\overline{\mathrm{N}}\left(\mathrm{r}, \frac{1}{\mathrm{f}^{\prime}-\lambda}\right)+\overline{\mathrm{N}}(\mathrm{r}, \mathrm{f})+\mathrm{S}(\mathrm{r}, \mathrm{f}) \\
\text { and } \mathrm{T}\left(\mathrm{r}, \mathrm{f}^{\prime}\right)<\overline{\mathrm{N}}\left(\mathrm{r}, \frac{1}{\mathrm{f}-\mu}\right)+\overline{\mathrm{N}}\left(\mathrm{r}, \frac{1}{\mathrm{f}^{\prime}-\lambda}\right)+\overline{\mathrm{N}}(\mathrm{r}, \mathrm{f})+\mathrm{S}(\mathrm{r}, \mathrm{f}) \text {. }
\end{array}
$$

Now, $\mathrm{f}^{\prime}-\lambda=\mathrm{f}-\lambda$

Hence, zeros of $f^{\prime}-\lambda$ occur only at the zeros of $f-\lambda$.

Therefore, $\mathrm{N}\left(\mathrm{r}, \frac{1}{\mathrm{f}^{\prime}-\lambda}\right)=\mathrm{N}\left(\mathrm{r}, \frac{1}{\mathrm{f}-\lambda}\right)$

Therefore, from (6) and (7), we have

$$
\mathrm{T}(\mathrm{r}, \mathrm{f})+\mathrm{T}\left(\mathrm{r}, \mathrm{f}^{\prime}\right)<2\left[\overline{\mathrm{N}}\left(\mathrm{r}, \frac{1}{\mathrm{f}-\mu}\right)+\mathrm{N}\left(\mathrm{r}, \frac{1}{\mathrm{f}-\lambda}\right)+\overline{\mathrm{N}}(\mathrm{r}, \mathrm{f})\right]+\mathrm{S}(\mathrm{r}, \mathrm{f})
$$

Hence using hypothesis, we have

$$
\begin{aligned}
& \mathrm{T}(\mathrm{r}, \mathrm{f})+\mathrm{T}\left(\mathrm{r}, \mathrm{f}^{\prime}\right)<\mathrm{S}(\mathrm{r}, \mathrm{f}) \\
& \text { or } 1 \leq \frac{\mathrm{S}(\mathrm{r}, \mathrm{f})}{\mathrm{T}(\mathrm{r}, \mathrm{f})+\mathrm{T}\left(\mathrm{r}, \mathrm{f}^{\prime}\right)} \rightarrow 0 \text { as } \quad \mathrm{r} \rightarrow \infty
\end{aligned}
$$

Thus, $1 \leq 0$ which is a contradiction.

This contradiction proves that $\lambda=\mu$.

Therefore, $\frac{\mathrm{f}-\mu}{\chi-\mu}=\frac{\mathrm{f}^{\prime}-\lambda}{\chi-\lambda}$

Hence the Theorem.

\section{References}

[1]. L. RUBEL and C. C. YANG, (1976) : Values shared by an entire function and its derivative in "Complex Analysis, Kentucky" (Proc. Conf.), Lecture notes in Mathematics, Vol. 599, pp. 101-103, Springer Verlag, Berlin 1977.

[2]. E. MUES and N. STEINMETZ (1979) : Mermorphic functionen, die mit ihrer Ableitung wert teilen. Manuscripta Math 29 (1979), 195-206.

[3]. G. JANK, E. MUES and L. VOLKMANN (1986) : Meromorphic functionen, die mit ihrer ersten und zweiten ableitung einen endlichen wert teilen, Complex variables 6, 51-71.

[4]. INDRAJIT LAHIRI (1998) : 'Uniqueness of meromorphic functions sharing the same 1-points'. Bull. Korean Math. Soc. 35, No. 2, pp. 375-385 